\title{
TOWARD COLLABORATIVE SCHEDULING
}

\author{
Ana Almeida, Goreti Marreiros \\ Knowledge Engineering and Decision Support Research Group - GECAD, \\ Computer Science Department \\ Institute of Engineering - Polytechnic of Porto \\ Porto, Portugal \\ \{ana,goreti\}@dei.isep.ipp.pt
}

\begin{abstract}
The scheduling process, usually involve the evaluation and selection of one alternative between a set of them. These decisions are not trivial, considering that they usually involve mitiple, and sometimes conflicting, criteria. Particularly in scheduling which aim is to find the trade off between loading efficiency and delivery accuracy laking into account holding costs, tardiness penalties and expedition charges. Scheduling decisions should be taken in respect with the result of the integration of different criteria weighted according the several perspectives from manufacturing environment namely, production, commercial, and quality. So, scheduling is a multi-criteria decision problem; in practice different schedulers may agree as to the key objectives but differ greatly as to their relative importance in ary given situation. The purpose of this paper is to address collaborative scheduling in complex dynamic manufacturing environment, presenting a collaborative scheduling approach which considers group decision support.
\end{abstract}

\section{INTRODUCTION}

Actual industrial environments deal with the globalization of the markets which caused a change in organizational perspective. Within the current scenario the organization tend to evolve to an organic system instead of a hierarchical one. The traditional hierarchical structure, functionality divided, is perceived as too rigid and slow, making it difficult to adapt quickly to changing environment requirements. There is a need to evolve to distributed structures with an horizontal functionality, where different functions collaborate in different processes.

Group decision-making represents an important role in actual organizations. The new economy demands that the decisions must be taken quickly however without damaging the quality of the decision-making process or its results. With the objective of making better decisions, more and more times decisions are taken by groups of individuals representing different organization perspectives, or even different organizations.

In the management field, most of the decisions we make involve the need to consult several persons discuss and argue for alternatives. For this reason Group

Please use the following format when citing this chapter: 
Decision Support Systems emerged as a very important area in the domain of Management, providing Collaborative Frameworks for Decision Support. Scheduling is one of the areas where this kind of systems make more sense, namely due to the complexity and dynamic nature of the environment, and the different kind of actors involved on the process.

Scheduling decisions are often characterized by goals, roles, activities and resources that are dynamically changing, or uncertain. For improve competitiveness scheduling decisions should arise from the integration of the different production functions where each participating actor should collaborate in achieving a solution.

This paper addresses the interaction between the scheduling actors through the integration of the different kinds of knowledge in a global view of the system and the potential synergy in association with the collaborative activity of those actors taking in account multiple criteria which can improve the scheduling process. Considering this fact the option for a collaborative approach using the concept of Group Decision Support System (GDSS) plays an important role.

This paper is organized as follows. Section 2 provides a general approach to the scheduling process, focusing some scheduling techniques and Collaborative Scheduling. In Section 3 is discussed the relation between group decision and scheduling and group decision support systems are presented. The proposed approach to collaborative scheduling through group decision support and its main features are described in Section 4. Finally Section 5 presents some conclusions.

\section{SCHEDULING}

Scheduling problems are among the most important in operations management because they impact the ability of manufactures to meet costumers' demands and make profit. The problem of scheduling can be considered as the allocation of a set of tasks to a limited number of resources, with an objective of satisfying constraints and a set of criteria (Baker, 1974).

Over the last fifty years a great deal of research has been focused on solving the scheduling problem, resulting in a wide variety of approaches. So, in the literature it is possible to find many contributions trying to solve the scheduling problem. Those contributions can be classified according to the nature of the obtained results or the used resolution approach. In respect to the nature of the obtained solution we can distinguish between optimization or approximation methods. According to the resolution approach there are individual and collaborative processes. We will focus on collaborative scheduling.

\subsection{Optimization Techniques}

In optimization strategies there are the so called efficient methods, which solve a given problem optimally. The complexity of these methods increases polynomially with respect to the size of the input, because all possible solutions are considered. These methods build an optimum solution from the problem data by following a simple set of rules which determine exactly the processing order. French (1982) predicts that no efficient algorithm will ever be developed for the majority of scheduling problems. 
Other kind of optimization strategies that find efficient solutions are the enumerative methods which generate schedules one by one using clever elimination procedures to verify if the non optimality of one schedule implies the non optimality of many others which were not yet generated, thereby preventing the need to search the complete space of feasible solutions. Here it can be included mathematical programming techniques, like the Mixed Integer linear Programming (Manne, 1960), Lagrangian Relaxation (LR) approaches (Fisher, 1973) and decomposition methods (Ashour, 1967), or Branch and Bound techniques (Lawler, 1973) where a dynamically constructed tree representing the solution space of all feasible schedules is implicitly searched. The great formulation difficulty and the excessive computational effort required can be partially suppressed through problems decomposition and introduction of relaxations in order to simplify the initial problem. Although these strategies are of tremendous theoretical value, the majority of them is unable to achieve feasible solutions to many problems and therefore is of limited practical use (Jain, 1999).

\subsection{Approximation Techniques}

Approximation techniques do not guarantee achieving exact solutions, but they are able to attain satisfactory, near optimal, solutions with a reduced amount of computational effort, therefore they are more suitable for larger problems. Here we can distinguish between constructive methods which build a complete solution from scratch using the problem data, or iterative methods which can modify one complete solution to another by continually reordering the sequence of operations.

On the first group it can be included some techniques that had a great success in scheduling problems resolution, due to their ease of implementation and reduced computational requirement (French, 1982), like Priority Dispatching Rules (Conway et al., 1967); and Bottleneck based heuristics, namely the Shifting Bottleneck procedure (Adams, 1988).

On the second group are the Local Search or Neighbourhood techniques (Aarts, 1997) which philosophy is a spatial search for valid better solutions within a certain amount of time. Some examples are: Tabu Search (Glover, 1989); Simulated Annealing (Kirkpatrick et al., 1983); and Genetic Algorithms (Goldberg, 1988). Some other strategies have been used like Constraint Satisfaction techniques (Fox, 1987) which aim reducing the effective size of the search space by applying constraints that restrict the order in which operations are sequenced and start times are assigned to each operation.

\subsection{Collaborative Scheduling}

Through complexity and fashion how production scheduling problems were tackled in the past, we can actually conclude that a great disparity exists between the way that scheduling systems solve problems and the way human resolves them. While automatic-scheduling systems need complete specification of goals and scenario before beginning problem resolution, persons progressively learn with scenario and change their goals during planning and execution. Besides that, there are different evaluation criteria, which are many times contradictory, arising from the diverse manufacturing perspectives involved in the scheduling process. Furthermore, actual 
industrial environments are often geographically dispersed; in the scheduling environment unplanned events occur frequently requiring scheduling decisions to be taken constantly.

There is the need of a diverse range of technical capabilities, usually representing different manufacturing perspectives to work together, sharing their knowledge through a collaboration process to arise to a global scheduling solution.

The determination of feasible and mutually-acceptable schedules can be a major challenge (Kowalczyk et al., 2004). Moreover, automated scheduling methods, whatever their nature is, might not produce realistic schedules in environments where contextual information is inadequately represented, objectives are complex and unstated, and situations are dynamic and uncertain. Such issues can be addressed by domain experts (Wiers, 1997; Almeida et al, 2002). So, under these circumstances, human schedulers bring to the scheduling process their inductive and pattern recognition abilities. This problem evidenced the necessity to create collaborative scheduling systems, where a group of users and scheduling engines collaborate in plans generation, identifying candidate alternatives, and selection one of them, thus profiting the better of the two worlds. This form of collaboration provides a very powerful approach to multi-attribute, multi-criteria, decision support in complex manufacturing environments.

There are some works on distributed, cooperative or collaborative scheduling. An example is the work developed by Kawamura and his colleagues (Kawamura, 2000) which is a distributed cooperative scheduling system, where several scheduling agents negotiate among them to realize schedule adjustments among busy departments. Another different approach is presented by Murthy and his colleagues (Murthy, 1997) where autonomous agents work together to produce a set of candidate alternatives, and a human scheduler make the final decision interacting with the other agents.

\section{GROUP DECISION}

\subsection{Scheduling and Group Decision}

One approach to tackle multi-criteria decision problems involves assigning weights to different criteria, aiming to come to a unique decision depending upon the assigned weights. In a collaborative decision making process, which frequently involves many people, experts on different aspects of the problem, all the relationships arising from the different departments representing the diverse manufacturing perspectives must be considered, so a set of weighted criteria seems to be the most adequate. For instance, from the manager point of view the most important criterion should be the profit and from a quality control department the most important criterion is product quality. But as economic conditions change, the relative importance of different criteria may change (Murthy, 1997). This requires users to modify these weighting factors periodically, by changing the relative importance of each criterion.

Architectures that enable collaboration are useful when it is not efficient or possible to perform a task by a single agent or human. They provide mechanisms which allow several users to contribute with their knowledge to the system, 
participating on an equal basis in the selection of candidate alternatives. So a collaborative framework capable of integrate multi-criteria decisions, arising from the different actors involved in the manufacturing process, can be most adequate.

One way of enhancing collaboration between agents and humans is to produce not one but many candidate solutions, evaluated with respect to multiple criteria. This allows users to gain important insights into the tradeoffs between multiple competing objectives. They express their preferences by imposing weighting factors for different criteria.

The benefits of group work are several: Groups are better than individuals at understanding problems; People are more responsible for decisions in which they participate; Groups are better than an individual participant at detecting flaws in proposed ideas; A group has more knowledge than any one member individually; Synergy may develop so that the effectiveness of the group is greater than what could have been produced individually; Working in a group could stimulate the group members and consequently the process of decision making; Participants' differing knowledge and processing skills allow results that could not be achieved individually.

If the there are big advantages associated to group work, there are also several dysfunctions related to this theme: Time consuming; High costs; Improper use of group dynamics; Tendency to rely on some members the most of the work; Tendency to make incomplete tasks analysis and to choose compromise solutions of poor quality.

\subsection{Group Decision Support Systems}

The Group Decision Support Systems (GDSS) aims to reduce the loss associated to group work and to maintain or improve the gain (Holsaple, 2001).

The term GDSS emerged effectively in the beginning of the eighty-decade. According to Huber (1984) a GDSS consists of a set of software, hardware, languages components and procedures that support a group of people engaged in a decision related meeting. A more recent definition is from Nunamaker et al. (1997) and says that GDSSs are interactive computer-based environment which support concerted and coordinated team effort towards completion of joint tasks.

Some of the advantages of using GDSS are: Equal and anonymous opportunity to contribute with ideas and opinions; Allows parallel communication between group members; Helps the facilitator of the meting in the schedule management; Eliminates too big domination of some group members in the meeting; Provides automatically organizational memory; Makes it possible, to find out the common and dissenting preferences among the group members.

In the 80's most of the research in the GDSS area was focused in the synchronous/same-place dimension, several decision rooms were configured. In the last years, with the proliferation of Internet the research on GDSS has its focus on the different-time/different place dimension. Several web-based GDSS have been developed (Marreiros et al, 2004; Dennis et al, 1996), and others like for instance the GroupSystems, that initially were developed just to support configuration of decision room type, are now able to support remote decision making.

As it was referred on introduction, the scheduling process, involve the evaluation and selection of one alternative between a set of them. This are not trivial decisions, 
because they usually involve multiple and conflicting criteria. Actual organizations are dispersed around the world. GDSS seems adequate to support the scheduling process.

\section{COLLABORATIVE SCHEDULING APPROACH}

A scheduling system should provide a user support, to assist him in build, change and revising processes of the scheduling plans and not deciding for him. The user has intelligence and knowledge acquired along the years that are not to underestimate. Nevertheless, a scheduling system could have autonomous capacity, suggesting alternatives according to some claimed criteria.

The user provides intuition, a notion about goals and appropriate trade-off, and refined problem resolution strategies. The computer provides skill to manage details, to assign and schedule resources and operations, and to analyze quantitatively the suggested choices.

This approach considers multiple scheduling objectives in a global multi-criteria collaborative framework. It generates several scheduling alternatives by using autonomous agents which encapsulates different scheduling algorithms. Each scheduling alternative represents a solution regarding an objective such as, accomplishment of deadlines, minimizing throughput times, maximizing profitability, product quality, and minimizing manufacturing disruptions. It provides decision support considering the negotiation process of a group of users, each one of them with a different perception of the problem, effectively acting as a team to achieve a common and unique solution.

The proposed approach, represented in Figure 1, includes two modules; the Scheduling Module responsible for the creation of a set of scheduling solutions and the Group Decision Support Module which is in charge of the selection of a scheduling solution. 


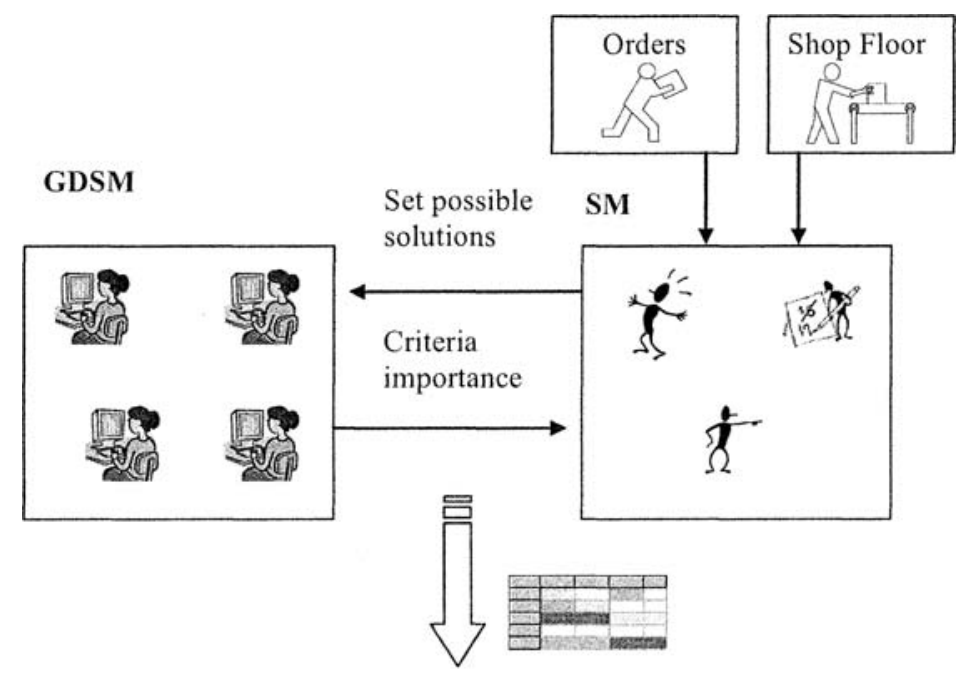

Schedulling Solution

Figure 1 - Approach to collaborative scheduling through group decision support

\subsection{Scheduling module}

Agents and multi-agent systems are an important area of research and development in Artificial Intelligence. Agents are typically knowledge representation entities characterized by independence and autonomy. The software agents are entities that have the ability to plan, to establish their actions ahead of time, to develop appropriated problems solving strategies, to communicate, or to share resources.

In this approach agents have the possibility to follow events as they occur in the environment, interpreting and sharing knowledge or data.

The Scheduling Module (SM) consists of multiple problem-solving methods (called agents) working at the same time on a common problem, so it does not represent any single method or heuristic, but is rather an attempt to use multiple techniques by encapsulating individual algorithms as autonomous agents.

The scheduling agents are different autonomous agents each one of them embodying a particular scheduling algorithm, as it was referred previously. According the scheduling criteria or objective, there is a broad range and variety of scheduling methods. One can apply any of the techniques referred on section 2, or any other algorithm or heuristic.

In this approach the information agent, in accordance with the type of scheduling problem, sets a time window for the generation of the several scheduling alternatives. Also some criteria are settled; this way just the agents embodying algorithms respecting the established criteria will be triggered. Only the alternatives 
generated within the settled time window are considered for analyse and discussion by the GDSM module.

The setting agent settle on the criteria importance according with the global preferences of the GDSM members, in order to cover all the relationships arising from the different departments.

\subsection{Group Decision Support module}

The Group Decision Support Module (GDSM) will support the members of a scheduling meeting and the facilitator. This last one prepares the meeting and invites a group of people to participate, and to exchange different points of view, expertise and information, in order to choice the "best" solution from the set of scheduling solutions proposed by the SM. After the generation of a set of alternative solutions, by the SM, the group members will use this module to individually choose the preferred one.

The GDSM is composed by the following components: Setup, Management, Argumentation, Multi-criteria, Voting and Database (Marreiros et al, 2004).

The facilitator of the meeting is supported by the GDSM in the execution of several activities: General schedule meeting configuration (specifying dates and time for the start and the end, as well as the goal of the meeting); Selection of participants, invitation sending, confirmation of reception and participant replacement, if necessary; Definition of the member importance (number of votes); Definition of decision rules (voting rules: consensus, majority, qualified majority, maximum number of voting cycles, anonymity; rules for argumentation: minimum/maximum number of arguments for each participant; the arguments are or are not visible to all the participants)

The participants of a group decision scheduling module are also supported by the GDSM: indication of the set of criteria; establishing individual preferences (will help the individual scheduler agent to ranking the several scheduling alternatives); support the exchange of arguments between group members (each participant will therefore argue for the most interesting alternatives or against the worst alternatives, according to his/her preferences). By expressing their arguments, participants expect to influence the others' opinions and make them change their choices.

\section{CONCLUSIONS}

The attention to the individual needs of each customer is a driving force behind many changes taking place in every industry sectors, so a scheduling decision, must take into account the knowledge and experience of different points of view, allowing the consideration of broad issues of the company rather than focusing on scheduling tasks for a single process. In a collaborative architecture multiple solutions can be generated and shared with the scheduling responsible for different stages of production and with customer service representatives, serving as basis for negotiating a set of solutions to come to the best alternative for the enterprise.

The presented approach addresses many of the limitations of existing job-shop scheduling systems, by providing a decision support collaborative framework for 
scheduling. It allows the evaluation of scheduling solutions by experts with different points of view, facilitating the incorporation of their knowledge into the system.

Improving schedule quality, lead to delivery accuracy, improved quality and fast time to market which results on a increase of competitiveness and profit. However, just as important are the opportunities to change business processes that the presented approach affords by showing multiple good alternatives, some of which may violate some constraints. These solutions can suggest good opportunities to look for alternative means of production and can suggest when it would be profitable to negotiate to change customer requirements or business policies. For instance if the deadlines imposed by a particular costumer are always tiny and can never be accomplished, it may indicate that it would be better to negotiate longer deadlines. So, significant savings and improved customer satisfaction are expected.

\section{ACKNOWLEDGEMENTS}

The authors would like to acknowledge FCT, FEDER, POCTI, POSI, POCI and POSC for their support to R\&D Projects and GECAD Unit.

\section{REFERENCES}

1. Aarts, E. and Lenstra, J. K. Local Search in Combinatorial Optimization, Wiley, Chichester, 1997

2. Adams, J., Egon B. and Zawack, D. The Shifting Bottleneck Procedure for Job Shop Scheduling. Management Science, Vol. 34, n $^{\circ} 3$ Março, USA, 1988.

3. Almeida, A; C. Ramos and C. Sílvio. Toward Dynamic Scheduling of Manufacturing. The International Journal for Manufacturing Science \& Production, Freund Publishing House, 2002.

4. Ashour, S. A Decomposition Approach for the Machine Scheduling Problem, International Journal of Production Research 6(2), 109-122, 1967

5. Baker, K. R. Introduction to Sequencing and Scheduling, John Wiley \& Sons, 1974.

6. Conway, R.et al. Theory of Scheduling, Addison-Wesley, Reading Massachusetts, 1967.

7. Dennis, P. et al. Using Internet to implement support for distributed decision Making. In Chapman \& Hall, UK, pp. 139-159, 1996.

8. Fisher, M. L., Optimal solution of scheduling problems using Lagrange multipliers: part I. Operations Research 21, 1114-1127, 1973

9. Fox, M. S. Constraint - Directed Search: A Case Study of Job-Shop Scheduling, Research Notes in Artificial Intelligence, Pitman Publishing, London, 1987

10. French, S. Sequencing and Scheduling - An Introduction to the Mathematics of the Job-Shop, Ellis Horwood, John-Wiley \& Sons, New York, 1982.

11. Glover, F .Tabu search - Part I", ORSA Journal on Computing, Vol. n'1, pp. 190-206, 1989.

12. Goldberg, D. Genetic Algorithms in Search Optimization and Machine Learning. Menlo Park, California, Addison-Wesley, 1988.

13. Holsapple, C.; Whinston, AB. Decision support systems: a knowledge-based approach. Thomson Learning, inc, 2001

14. Huber, G. Issues in the design of group decision support systems. Mis Quarteley, 3(8), 1984.

15. Jain, A. S. and Meeran, S. Deterministic job-shop scheduling: Past, present and future. European Journal of Operational Research $113390-434,1999$

16. Kawamura, $T$ et al. Development of a Distributed Cooperative Scheduling System Based on Negotiations between Scheduling Agents, In Systems and Computers in Japan, vol. 31, $\mathrm{n}^{\circ} 1,2000$.

17. Kirkpatrick, S.;Jr. C. Gellat and M. Vecchi. Optimisation by Simulated Annealing", Science, 220 (4598) pp. 671-680, 1983

18. Kowalczyk, R.et al..Towards supporting collaborative scheduling in adaptive supply networks with negotiation agents. Proc. Theory and Applications of e-Negotiations, Poznan, Poland, 2004. 
19. Lawler, E. L. Optimal Sequencing of a Single Machine Subject to Precedence Constraints, Management Science, vol 19, 544-546, 1973.

20. Manne, A. S. On the Job-Shop Scheduling Problem, Operations Research, vol 8, 219-223,1960.

21. Marreiros, J. P. Sousa and C. Ramos, "WebMeeting - a group decision support system for multicriteria decision problems". ICKEDS04, 2004.

22. Murthy, S. et al. Agent-based cooperative scheduling, In AAAI97 Workshop on Constraints and Agents., pages 112--117, 1997

23. Nunamaker, J.F. et al. Lessons from a dozen years of group support systems research: A discussion of lab and field findings, Journal of Management Information Systems, 13 (3), 1997.

24. Wiers, V. Human-Computer Interaction in Production Scheduling: Analysis and Design of Decision Support Systems for Production Scheduling Tasks, Ph.D. Thesis, Eindhoven University of Technology, Holland, 1997. 